http://dx.doi.org/10.18778/0208-6107.32.12

\author{
Gerhard Schönrich \\ Institut für Philosophie \\ Technische Universität Dresden \\ gerhard.schoenrich@tu-dresden.de
}

\title{
WAS MACHT ENTSCHEIDUNGEN ZU WEISEN ENTSCHEIDUNGEN?
}

\begin{abstract}
Zusammenfassung
Der Begriff einer weisen Entscheidung ist methodisch leichter zugänglich als der allgemeine Begriff der Weisheit. Wenn wir wissen, was weise Entscheidungen ausmacht, dann öffnet sich uns auch ein Zugang zu den entsprechenden Eigenschaften und Fähigkeiten einer Person. Die Abhandlung soll zeigen, dass sich eine Analyse weiser Entscheidungen nicht auf eine attributive Lesart: „S entscheidet weise“ beschränken kann, sondern die adverbiale Lesart: „S entscheidet sich auf eine weise Art" berücksichtigen muss. Die externalistische Perspektive der ersten Lesart führt zwar zu einer notwendigen qualitativen Bedingung (langfristige Verbesserung der Situation für möglichst viele Beteiligte), der jede weise Entscheidung im Resultat gerecht werden muss. Erst die internalistische Perspektive der zweiten Lesart entspricht unseren Intuitionen, dass ein solches Resultat nicht zufällig zustande gekommen sein darf, sondern sich bestimmten epistemischen Leistungen des Akteurs verdankt, wie seiner Urteilskompetenz und Reflexionsfähigkeit der Gründe für die Entscheidung. Diese Leistungen schließen insbesondere die Meta-Fähigkeit, erststufige Gründe von zweitstufigen Gründen zu trennen ein, die sich nicht auf den Wert, den das Objekt der Entscheidung hat beziehen, sondern auf den Wert der Einstellung, die der Entscheidung zugrunde liegt.
\end{abstract}

\section{Schlüsselwörter:}

Weisheit, Entscheidung, Gründe, Wert

\section{BEMERKUNGEN ZUR METHODE}

Das Thema „Weisheit hat in den letzten zwei Jahrzehnten vor allem in der psychologischen Forschung eine Renaissance erlebt. ${ }^{1}$ Die Frage „Was ist Weisheit?" wird hier meist mit einer Intuition darüber beantwortet, worin Weisheit besteht, und mit der Beschreibung von Eigenschaften erklärt, die weise Personen

\footnotetext{
${ }^{1}$ Für eine kurze Darstellung der Geschichte der Weisheitsforschung vgl. Judith Glück, Weisheit. Die fünf Prinzipien des gelingenden Lebens (München: Kösel-Verlag, 2016), 13 ff.
} 
besitzen müssen, damit ihnen das Prädikat „weise“ zugeschrieben werden kann. Weisheit sei breites und tiefes Wissen über die grundlegenden Themen menschlichen Lebens. Der Weise, der über solches Wissen verfüge, sei sowohl reflektiert (der Grenzen seines Wissens bewusst), als auch verpflichtet auf die Vertiefung vorhandenen und den Erwerb weiteren Wissens. Zugleich bewährt sich der Weise in der Kunst einer rationalen Lebensführung (im Umgang mit den eigenen Gefühlen und mit den Mitmenschen). ${ }^{2}$

Der so charakterisierte Weise müsse bestimmte Charaktereigenschaften besitzen. Genannt werden z. B. Offenheit, Emotionskontrolle, Einfühlungsvermögen, Reflektivität im Sinne kritischen Hinterfragens und realistischer Einschätzung der eigenen Möglichkeiten. Wie zuverlässig ist der Schluss von den konzeptualisierten Intuitionen über Weisheit auf die kognitiven und praktischen Eigenschaften oder Fähigkeiten der weisen Person? Dieses methodische Vorgehen leidet an einer Unklarheit, die auf eine fehlende Unterscheidung zurückgeht:

- die Eigenschaft, weise zu sein, ist ein Merkmal, das die eine Person weise macht, die andere Person nicht.

- der Begriff der Weisheit wird gebraucht, wenn wir über die Eigenschaft, weise zu sein, nachdenken und Forschungen dazu anstellen.

Natürlich geht es auch Philosophen um die Eigenschaft weise zu sein (bzw. um das Bündel von Eigenschaften und Fähigkeiten, die Weisheit ausmachen). Warum sollten wir nicht gleich mit einer Untersuchung dieser Eigenschaft anfangen? Die Antwort ist: wir brauchen den Begriff "Weisheit" schon um die entsprechende Eigenschaft, um die es uns geht, bei Personen zu identifizieren. Möglicherweise bezeichnet das Wort „weise“ oder „Weisheit“ ja mehrere unterschiedliche Begriffe. Die beste Methode, einen Begriff zu untersuchen, besteht immer noch darin, die linguistischen Daten heranzuziehen und genauer hinzuschauen, wie wir reden. Manchmal drücken zwei verschiedene Worte denselben Begriff aus, manchmal ist es auch so, dass ein und dasselbe Wort verschiedene Begriffe ausdrückt. Hier ist also Vorsicht geboten.

Wenn wir als Philosophen den Begriff der Weisheit analysieren, dürfen wir erwarten, dass wir einige begriffliche Wahrheiten über die Eigenschaft, weise zu sein, herausfinden, d. h. einige Wahrheiten, die schon durch die Natur des Begriffes gegeben und garantiert sind. Kandidaten für solche begriffliche Wahrheiten sind:

1. Weisheit ist ein normativer Begriff.

2. Weisheit hat nicht nur internalistische, sondern auch externalistische Aspekte.

\footnotetext{
${ }^{2}$ In den Intuitionen unterscheiden sich Philosophen und Psychologen kaum. Vgl. z. B. Sharon Ryans „Deep Rationality Theory“ in: Sharon Ryan, „Wisdom, Knowledge and Rationality,” Acta Analytica 27, Nr. 2 (2012) mit Glücks Konzept „Weisheit,” 28.
} 
Diesen Fokus auf den Begriff der Weisheit können wir noch enger fassen, indem wir nicht Weisheit generell, sondern zunächst weise Entscheidungen oder weise Ratschläge untersuchen. Der Begriff einer weisen Entscheidung ist leichter zugänglich als der allgemeine Begriff der Weisheit. Die Leitidee für diesen methodischen Zugang ist: Wer in den meisten Fällen weise Entscheidungen trifft, ist eine weise Person, d. h. hat die Charakter-Eigenschaft, weise zu sein. Wenn wir schließlich wissen, was weise Entscheidungen ausmacht, dann öffnet sich uns auch ein kontrollierter Zugang zu den entsprechenden Eigenschaften und Fähigkeiten einer Person.

$\mathrm{Zu}$ 1) Warum ist der Begriff der Weisheit bzw. der Begriff einer weisen Entscheidung normativ?

1. Wer in einer schwierigen Entscheidungssituation steht, soll möglichst gut, am besten aber möglichst weise entscheiden. Weisheit ist nicht nur ein gradueller Begriff, sondern - jedenfalls in Entscheidungssituationen - auch mit einem Sollen verbunden - durchaus ähnlich einem moralischen Sollen. Wir machen nicht nur Personen Vorwürfe, die moralisch falsch gehandelt haben, sondern auch Personen, die töricht entschieden haben. Das tun wir, weil wir in diesem Fall von einer epistemischen und praktischen Fehlleistung ausgehen, die der Akteur zu verantworten hat. Im Unterschied zu moralischen Verfehlungen, denen wir mit Sanktionen begegnen, finden wir den Toren aber eher lächerlich und bedauernswert. Das scheint ein wichtiger Unterschied zu sein. Wie auch immer - wer in einer schwierigen krisenhaften Situation steht, soll möglichst weise entscheiden. Diese normative Dimension ist bisher unerforscht. Wir klammern sie hier aus.

2. $\mathrm{Zu}$ sagen, man habe selbst eine weise Entscheidung getroffen oder einen weisen Rat erteilt, oder die Zuschreibung einer weisen Entscheidung oder weisen Ratschlags an andere, ist eine Redehandlung, die im performativen Teil nicht nur konstatierend ist, sondern auch eine Pro-Einstellung des Billigens und Anerkennens ausdrückt. Von einer Entscheidung oder einem Rat mit schlechten Folgen sagen wir nicht, dass sie „weise“ sei; von einer Entscheidung mit guten Folgen für uns oder für (viele) andere dagegen schon. Ebenso reden wir nicht von einer ,weisen Entscheidung“, wenn in einer banalen Alltagssituation (,Soll ich lieber ein Eis essen oder ein kühles Bier trinken?") eine Wahl getroffen wird. Die Redehandlung muss sich schon auf eine schwierige krisenhafte Situation beziehen, in der eine Wahl mit weitreichenden Folgen getroffen wird. Wenn jemand durch seine Entscheidung oder durch seinen Rat eine solche Situation meistert, dann zollen wir ihm mit der Zuschreibung ,weise“ auch Lob und Anerkennung. Die evaluative Komponente solcher Redehandlungen bezieht sich auf: 
- hinreichend positive Folgen der Entscheidung: idealerweise wird das in der jeweiligen Situation Bestmögliche gewählt;

- auf die kognitiven (und ggf. praktischen) Leistungen, die für eine rationale Wahl in einer komplexen und bedeutsamen Situation erbracht wurden.

$\mathrm{Zu}$ 2) Internalistische und externalistische Aspekte des Weisheitsbegriffs.

Die attributive Verwendung des Ausdrucks „weise“ in „eine weise Entscheidung / weiser Rat" stellt auf das Resultat der Entscheidung ab. Die adverbiale Verwendung in „Er entscheidet sich weise (auf weise Art)“ dagegen stellt auf den Entscheidungsprozess ab. Die attributive Verwendung lässt eine rein externalistische Betrachtungsweise zu, die adverbiale Verwendung erfordert dagegen eine internalistische Betrachtungsweise. Statt von attributiver oder adverbialer Verwendung zu sprechen, können wir auch das Raster „resultatorientiert“ und „prozessorientiert" benützen:

- Resultatorientiert: Es ist für S in Situation C weise, sich zu entscheiden, $\mathrm{H}$ zu tun;

- Prozessorientiert: S entscheidet sich in Situation C weise, $\mathrm{H}$ zu tun.

Vor der Frage nach dem, was eine Entscheidung zur weisen Entscheidung macht, steht noch die Frage: Was ist eine Entscheidung? Nach Heckhausen / Gollwitzer ist die Psychologie von Entscheidungen durch vier (idealtypische) Phasen gekennzeichnet: ${ }^{3}$

1. In der prädezisionalen Phase wird eine Bestimmung des Ziels vorgenommen. Sie mündet nach mehr oder weniger gründlicher Abwägung (der Wünsche und ihrer erwartbaren Realisierung sowie deren Folgen und im Abgleich mit Wertvorstellungen) in der Bildung einer Intention. Die Festlegung auf ein Ziel (Willensbildung) bildet den Kern der Entscheidung.

2. Nach dem festgelegt wurde, was erreicht werden soll, wird in der präaktionalen Phase geplant, wie das Ziel realisiert werden soll, welche Handlung das geeignetste Mittel zur Erreichung des Ziels ist. Hier können die Umstände spezifiziert werden, unter denen die Handlung durchgeführt werden soll (ggf. nicht durchgeführt werden soll).

3. In der aktionalen Phase schließlich wird die Handlung initiiert. Die Handlungsausführung ist auf das Ziel ausgerichtet und wird im Sinne einer fortlaufenden Kontrolle bei unvorhergesehenen Umständen angepasst.

4. Die Psychologen identifizieren auch noch eine postaktionale Phase, in der nach der Deaktivierung der Intention die Zielerreichung (oder das Verfehlen des Ziels ggf. auch eine Modifikation) bewertet wird (Soll-Ist-Vergleich,

\footnotetext{
${ }^{3}$ Heinz Heckhausen und Peter M. Gollwitzer, „Thought Contents and Cognitive Functioning in Motivational versus Volitional States of Mind," Motivation and Emotion 11, Nr. 2 (1987): 101-120.
} 
Beurteilung der Entscheidungsqualität). Diese Phase wird als wichtig für die Lerneffekte und die Herausbildung von Entscheidungs-Routinen betrachtet. Diese Phase spielt für unsere Überlegungen hier keine Rolle.

Nun sollten sich weise Entscheidungen von normalen oder bloß klugen Entscheidungen durch eine besondere Qualität auszeichnen. Das wesentliche Merkmal einer weisen Entscheidung oder Handlung besteht darin, solche Ziele umzusetzen, welche die zu erwartende Situation langfristig verbessern. Damit nehmen wir ein Motiv stoischer Weisheitslehren auf, die den therapeutischen Charakter der praecepta sapientium betonen: So wie die Medizin den Leib in einen besseren Zustand führt, so sollen die Anweisungen des Weisen die Kräfte der Seele aktivieren. ${ }^{4}$ Nach unserem Vorschlag betrifft eine Verbesserung der Situation aber nicht nur mentale Aspekte (wie z. B. die generellen Handlungsabsichten der Akteure und die von ihnen verfolgten Ziele), sondern auch epistemische (wie z. B. die Verbesserung von Erkenntnis- und Forschungsmöglichkeiten), und natürlich auch praktische Aspekte. Versuchen wir diese Beschreibung mit Blick auf „weise“ als Analysandum in ein Analyseschema zu bringen: ${ }^{5}$

Es ist für $\mathrm{S}$ in Situation C weise (sich zu entscheiden) $\mathrm{H}$ zu tun gdw.

(i) S das Ziel Q erreichen möchte [Handlungsbedingung];

(ii) die Erreichung von $\mathrm{Q}$ eine langfristige Verbesserung der erwarteten Situation $\mathrm{C}^{*}$ involviert, die nicht nur $\mathrm{S}$, sondern einer hinreichend großen Menge von weiteren Individuen zugutekommt [qualitative Bedingung];

(iii) $\mathrm{H}$ für $\mathrm{S}$ in $\mathrm{C}$ das geeignetste Mittel ist, um $\mathrm{Q}$ zu erreichen [Bedingung der Rationalität];

(iv) $\mathrm{S}$ Gründe hat (sich zu entscheiden), $\mathrm{H}$ zu tun [Rechtfertigungsbedingung].

Bei adverbialen oder prozessorientierten Zuschreibungen muss die Rechtfertigungsbedingung stärker gemacht werden, indem verlangt wird, dass der Akteur nicht nur wie nach der attributiven resultatorientierten Lesart überhaupt Gründe für seine Entscheidung hatte (nämlich, dass H die vorliegende Situation langfristig verbessern wird), sondern dass die Entscheidung des Akteurs auch tatsächlich auf diesen Gründen basiert und der Zugang zu diesen auch qualitativ ausreichend war. Diese Perspektive wollen wir hier weiterentwickeln:

Wir nehmen die Bedingung (i) und (ii) zum Ausgangspunkt, die sich für eine strikt resultatorientierte Betrachtung eignen, um dann zu fragen, ob die Entscheidung für $\mathrm{H}$ nicht auch durch einen glücklichen Zufall hätte so zustande

\footnotetext{
${ }^{4}$ Cicero Tusculanae Disputationes III, 5 f. IV, 23 ff. Zu weiteren Belegen, vgl. Christoph Horn, Antike Lebenskunst. Glück und Moral von Sokrates bis zu den Neuplatonikern (München: C.H. Beck, 1998), 16 und Art. Andreas Speer, „Weisheit,” in Historisches Wörterbuch für Philosophie, hg. von Joachim Ritter, Bd. 12 (Basel: Schwabe AG, 1971), 373.

${ }^{5}$ Das (hier leicht modifizierte) Analyseschema geht auf ein Konzept zurück, das in der Arbeitsgruppe des DFG finanzierten Dresdner Projekts „Eine mehrdimensionale Theorie der Weisheit“ entwickelt wurde.
} 
kommen können und welche weitere Bedingung diesen Zufall ausschaltet. So werden wir gezwungen, den Ausgangspunkt durch immer weitere Bedingungen zu ergänzen und die externalistische resultatorientierte Analyse schrittweise durch eine internalistische prozessorientierte Analyse zu ersetzen.

\section{TERMINOLOGISCHE KLÄRUNGEN}

1. Im Folgenden soll mit dem Ausdruck „weise Entscheidung“ auch der Fall eines weisen Ratschlags mitgemeint sein. $\mathrm{Ob}$ ich mich weise für eine bestimmte Handlung entscheide, oder ob ich jemandem, der vor einer Entscheidung steht, einen Ratschlag gebe, wie er sich entscheiden soll, kann in den Hinsichten, die hier relevant sind, als äquivalent betrachtet werden.

2. In der Analyse von Entscheidungen geht es primär um den epistemischen Aspekt, d. h. hier um die kognitiven Ressourcen, die wir gebrauchen müssen, um zu weisen Entscheidungen zu kommen. Es geht nicht um den praktischen Aspekt einer gelingenden Lebensführung, wenn wir fragen, wie ein gutes Leben zu führen sei - auch wenn vermutet werden darf, dass die Kunst, ein gelingendes Leben zu führen, neben der Emotionskontrolle (die hier nicht thematisch ist), wesentlich von diesen kognitiven Ressourcen abhängt. Der praktische Aspekt fehlt auch nicht gänzlich. Er wird nur viel weiter gefasst als in der Frage nach der Kunst einer guten Lebensführung und kommt in der qualitativen Bedingung zum Ausdruck. Die Entscheidung muss im Resultat ja zu einer Verbesserung der Situation führen. Entscheidungen zielen auch hier auf das praktisch Gute. Die Frage, die wir hier in den Vordergrund stellen, zielt auf die epistemischen Anforderungen, die solche (auf das praktisch Gute zielenden) Entscheidungen notwendigerweise erfüllen müssen, um als weise zu gelten.

3. Im Folgenden müssen wir uns nicht auf eine bestimmte Handlungstheorie festlegen. Die Formulierungen:

- Der Akteur S vollzieht die Handlung H, um Q zu realisieren (teleologisch)

- Der Akteur S tut H in der Absicht Q (intentional)

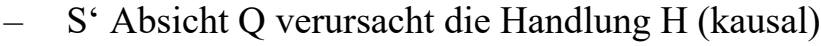

können für unsere Zwecke als äquivalent betrachtet werden. Mehr als eine liberal verstandene Standard-Theorie brauchen wir hier nicht. Sie lässt sich in drei Punkten umreißen:

(1) $\mathrm{S}$ entscheidet sich für eine Handlung $\mathrm{H}$ heißt: $\mathrm{S}$ hat eine konative Pro-Einstellung (Q) und eine kognitive Einstellung (Überzeugung), dass $\mathrm{H}$ das geeignetste Mittel zur Erreichung von $\mathrm{Q}$ ist.

(2) Konative Pro-Einstellungen: S hat die Absicht, den Wunsch, Willen, das Ziel Q 
(3) Das Paar aus konativer Einstellung und kognitiver Einstellung ist der Primärgrund dafür, dass S H tut.

Auch die Unterschiede zwischen den konativen Pro-Einstellungen: Absicht, Wille, Wunsch, Ziel usw. dürfen wir für unsere Zwecke übergehen. S will Q, möchte $\mathrm{Q}$, wünscht $\mathrm{Q}$ oder strebt nach $\mathrm{Q}$ wird als äquivalent behandelt. Solche konativen Einstellungen sind ebenso wie die kognitive Einstellung der Überzeugung propositionale Einstellungen. Beide Einstellungen bilden ein Paar, das den Primärgrund für die Handlung $\mathrm{H}$ bildet. Ein bloßer Wunsch ohne die begleitende Überzeugung von einem Mittel ist kein Primärgrund, der entscheidungsrelevant oder handlungswirksam ist.

Im Folgenden geht es vor allem um das erste Glied in dem Paar, nämlich um den Grund einer Handlung. Dass ich $\mathrm{H}$ tue und nicht non-H liegt daran, dass ich den Wunsch nach Q oder das Ziel Q habe. Gründe G sind entweder externe oder interne Tatsachen (wie Wünsche, Ziele usw.) Sie spielen eine dreifache Rolle:

(a) $\mathrm{G}$ rechtfertigt die Handlung $\mathrm{H}$ in dem Sinne, dass $\mathrm{G}$ diese Handlung $\mathrm{H}$ als richtig erweist (normative Rolle).

(b) G motiviert die Ausführung von $\mathrm{H}$, d. h. G ist der Grund, weshalb S H tut (psychologische Rolle).

(c) G erklärt, warum S H tut (rationale Rolle).

In der Analyse von Entscheidungen steht (a) im Vordergrund, solange es um die Rechtfertigungsbedingung geht, (b) und (c), wenn es um die Rationalitätsbedingung geht.

\section{EIN MODELLFALL}

(Bürgermeister-Szenario 1):

1) Dem Bürgermeister $S$ wird aus EU-Mitteln eine sehr hohe Fördersumme für den insolvenzbedrohten Haushalt der Gemeinde angeboten, wenn er in seiner Gemeinde ein Gewerbegebiet einrichtet.

2) Gegen das Gewerbegebiet hat er starke Vorbehalte. S wünscht sich aber, das Gewerbegebiet einrichten zu können (bzw. den Gemeinderat dazu zu bringen, die Einrichtung des Gewerbegebiets zu beschließen), um die Fördersumme zu erhalten.

3) Für das Gewerbegebiet spricht, dass langfristig Gewerbesteuereinnahmen zu erzielen sind, aus denen das lange geplante Kindergartenprojekt finanziert werden kann.

4) Für das Gewerbegebiet müsste aber ein nahes Waldgebiet abgeholzt werden, das entscheidend zur Wohn- und Luftqualität im Ort beiträgt. Die Abholzung würde zu Einbußen im Tourismus-Geschäft führen.

Nehmen wir an, der Bürgermeister S entscheidet sich für das Gewerbegebiet. Ist die Entscheidung des Bürgermeisters weise? 
Bevor wir uns fragen, wie der Bürgermeister zu seiner Entscheidung kommt, d. h. welche Überlegungen er anstellt und welche kognitiven Leistungen er dabei erbringt, können wir einfach nur von außen auf das Ergebnis der Entscheidung schauen. Tatsächlich erreicht der Bürgermeister bzw. der Gemeinderat mit der Entscheidung eine große Verbesserung der Situation der Gemeinde. Die Insolvenz ist abgewendet und der Kindergarten kann aus den Gewerbesteuereinnahmen finanziert werden.

Die leitende Intuition ist: Eine Entscheidung kann nur dann weise sein, wenn sie eine schwierige Situation größtmöglich und langfristig verbessert. S tut im Ergebnis das Bestmögliche in dieser schwierigen Situation.

Was auch immer wir über weises Entscheiden in einer internalistischen Betrachtung noch herausfinden, es impliziert folgende Bedingung:

(WE-0) S entscheidet sich in Situation $\mathrm{C}$ weise, $\mathrm{H}$ zu tun nur dann, wenn

(i) $\mathrm{H}$ unter den Handlungsalternativen, die $\mathrm{S}$ in $\mathrm{C}$ zur Verfügung stehen, das geeignetste Mittel ist, das zur Erreichung des Ziels Q, nämlich zur größtmöglichen und langfristigen Verbesserung der zu erwartenden Situation $C^{*}$ beiträgt (qualitative Handlungsbedingung).

In einer externalistischen Betrachtung kommen wir nicht weiter als bis zu dieser notwendigen Bedingung für eine weise Entscheidung. Ist sie auch hinreichend? Wohlgemerkt wir fragen nicht, ob sie auch hinreichend für die Analyse des Entscheidungsprozesses ist - das ist sie natürlich nicht. Ist sie hinreichend für das resultatorientierte Zuschreiben einer weisen Entscheidung?

Es wäre eine empirische Überprüfung wert, herauszufinden, wie viele Leute zögern, hier schon von einer weisen Entscheidung zu sprechen und was der Grund ist, der uns hindern könnte, S eine weise Entscheidung zuzuschreiben.

Um den Punkt schärfer herauszuarbeiten, ergänzen wir das BürgermeisterSzenario mit folgender Modifikation:

\section{(Bürgermeister-Szenario 2):}

Angesichts der Handlungsalternative $\mathrm{H}$ vs. non-H würfelt der Bürgermeister S. Bei gerader Augenzahl will S H tun, bei ungerader non-H. S würfelt eine gerade Augenzahl und entscheidet sich damit für das Gewerbegebiet.

Die Gemeinde hat in diesem Szenario einfach Glück gehabt, dass das Ergebnis so günstig ausgefallen ist. Mit einer Wahrscheinlichkeit von 0.5 hätte sich die Situation auch nicht verbessern können. Die meisten von uns haben hier wohl die starke Intuition, dass eine so zustande gekommene Entscheidung nicht weise zu nennen ist. Man mag es immer noch eine gute Entscheidung nennen, weil das Ergebnis gut für die Gemeinde ist, egal wie es zustande gekommen ist.

Gegen (WE-0) als erschöpfende Definition einer weisen Entscheidung sprechen drei Argumente: 
1. Mit (WE-0) werden wir der evaluativen Komponente unserer $\mathrm{Zu}$ schreibungspraxis nicht gerecht. Unser Lob zielt nicht allein auf das gute Resultat, sondern auch auf das Zustandekommen der Entscheidung. Wir finden es gut, wenn es nach langer Dürre regnet. Hier loben wir das gute Ergebnis, aber nicht das Zustandekommen des Regengusses. Hätte das Tiefdruckgebiet eine andere Bahn genommen, würde die Dürre noch andauern. Wenn wir jemandem eine weise Entscheidung zuschreiben, wollen wir auch sagen, dass der Entscheider an dem Ergebnis durch eine Leistung beteiligt ist. Die evaluative Komponente in unserer Zuschreibungspraxis zwingt uns also, über (WE-0) hinauszugehen.

2. Würden wir bei (WE-0) stehen bleiben, kämen wir nie zu der personalen Eigenschaft „weise“. Wenn es auch ein Zufallsgenerator sein kann, der zu guten Ergebnissen führt, erübrigt sich die Frage nach personalen Eigenschaften wie z. B. besondere kognitive Fähigkeiten, die für ein gutes Resultat verantwortlich sind.

3. Der Begriff der Entscheidung hat Implikationen, die wir ignorieren würden, wenn wir auch zufällig zustande gekommene Ergebnisse akzeptieren würden. Eine notwendige Bedingung für eine Entscheidung ist, dass $\mathrm{S}$ eine Wahl zwischen einer Alternative haben muss. Die Wahl durch einen Zufallsgenerator zustande zu bringen, hieße gegen die RationalitätsAnnahme zu verstoßen. Wir würden S nicht als rationalen Akteur verstehen, wenn nicht S (d. h. S's Wünsche oder Absichten), sondern ein Zufallsgenerator Urheber der Entscheidungen wäre.

Ein erster Schritt über (WE-0) hinaus ist das

Postulat: S entscheidet sich weise, wenn die Entscheidungsfindung auf die $S$ zur Verfügung stehenden kognitiven Ressourcen zurückzuführen ist.

Mit dem Postulat wechseln wir von der externalistischen Sichtweise in eine internalistische prozessorientierte Sichtweise. Dabei ist der Ausdruck „kognitive Ressourcen" nur ein Platzhalter für ein ganzes Bündel von genauer zu untersuchenden kognitiven Fähigkeiten, Überzeugungen, Wissen und Verstehensleistungen, über das S verfügen muss. Im Fall der Leistung eines Sportlers geben wir uns auch nicht mit der Erklärung zufrieden, dass das überragende Ergebnis auf die sportlichen Ressourcen des Akteurs zurückzuführen sei. Wir wollen wissen, welche Trainingsmethoden, welche körperliche Konstitution, welche spezifischen körperlichen und mentalen Fähigkeiten wie Zustand der Muskeln, Konzentration, Taktik usw. die Leistung erklären können.

Welche internen Faktoren, sind an der Entscheidungsfindung beteiligt? So wie wir das Bürgermeister-Szenario beschrieben haben, sind es die konativen Pro-Einstellungen wie Wünsche bzw. Absichten oder Ziele und die Überzeugungen von den Mitteln, wie die Absicht umzusetzen bzw. das Ziel zu erreichen ist. Dieses Paar aus einer konativen Pro-Einstellung mit Inhalt Q und der Überzeugung zu $\mathrm{H}$ als Mittel ist der Primärgrund für S zu H-en. 


\section{EIN MINIMALES KONZEPT FÜR WEISES ENTSCHEIDEN}

Die Entscheidungsfindung erfolgt mit Hilfe von Überlegungen, die sich auf Gründe stützen. Der primäre Grund für die Entscheidung des Bürgermeisters zugunsten des Gewerbegebiets scheint der Wunsch nach der Fördersumme zu sein. Es ist dieser Wunsch oder dieses Ziel, das zusammen mit der Überzeugung von der Geeignetheit des Mittels, die Entscheidung rechtfertigt. Wohlüberlegt sind Entscheidungen, die sich in der Suche nach dem Bestmöglichen auf rechtfertigende Gründe stützen. Es geht also nicht nur darum, in einer schwierigen Situation das Bestmögliche zu tun, sondern überlegt das Bestmögliche zu tun.

(WE-min) $\mathrm{S}$ entscheidet sich in Situation $\mathrm{C}$ weise, $\mathrm{H}$ zu tun gdw.

(i) $\ldots$

(ii) $\mathrm{S}$ beabsichtigt $\mathrm{Q}$ zu realisieren und $\mathrm{S}$ die Überzeugung hat, dass $\mathrm{H}$ das geeignetste Mittel ist, Q zu erreichen (internalistische Handlungsbedingung).

Dass die Entscheidung für $\mathrm{H}$ zufällig zustande kommt, diese Art Zufall schalten wir mit der Bedingung (ii) aus. S ist ein Akteur, der mit $\mathrm{H}$ ein Ziel realisieren will, und eine Überzeugung von der Umsetzung dieses Ziels hat. Was wir mit „überlegt" meinen, geht jedoch über die Abwendung von Zufälligkeit hinaus. Wir meinen damit, dass sich die Handlung im Lichte des Ziels als richtig oder gerechtfertigt erweist. Gegeben, dass S das Ziel Q verfolgt, ist $\mathrm{H}$ richtig. Gefragt, warum S H tut, würde S antworten, weil es richtig ist, im Hinblick auf Q $\mathrm{H}$ zu tun.

(iii) das Ziel-Überzeugungs-Paar nach (ii) der rechtfertigende Primärgrund G für S's H-en ist (Rechtfertigungs-Bedingung).

Nun könnte unser Bürgermeister zwar Q anstreben und $\mathrm{H}$ für das beste Mittel, Q zu erreichen halten, aber dennoch $\mathrm{H}$ aus einem ganz anderen Grund tun wollen. Er könnte das Gewerbegebiet nicht wegen der hohen Fördersumme, sondern z. B. wegen eines Freundes einrichten wollen, der sich mit seiner Firma in der Gemeinde ansiedeln möchte und ihm eine Bestechungssumme zahlt. In externalistischer Betrachtung verhält sich der Bürgermeister konform zu Bedingung (iii): er ist in externalistischer Sicht in seiner Entscheidung auch gerechtfertigt. Es gibt diesen normativen Rechtfertigungsgrund immer noch - nur ist es eben nicht der Grund, aus dem der Bürgermeister die Handlung vollzieht.

Wäre da nicht der Amigo mit dem Bestechungsgeld gewesen, hätte der Bürgermeister eine andere Entscheidung getroffen. Es hätte unter den bisher festgelegten Bedingungen leicht sein können, dass sich der Bürgermeister anders entschieden hätte. Nur zufällig stimmt die Entscheidung mit allen bisherigen Bedingungen (i) bis (iii) überein. 
Es geht hier nicht um ein moralisches Argument. Natürlich ist Bestechung verwerflich. Und weise Entscheidungen sollten auch moralische Bedingungen erfüllen. Hier geht es aber darum, die Möglichkeit auszuschalten, dass das H- tun zufällig mit dem rechtfertigenden Grund G konform geht. Um das sicherzustellen, müssen wir fordern:

(iv) S H aus dem Primärgrund G ausführt (starke Rationalitätsbedingung).

Die starke Rationalitätsbedingung schließt die schwache Rationalitätsbedingung ein, dass S sich konform zu G verhält.

Machen wir eine Bestandsaufnahme: $\mathrm{S}$ tut $\mathrm{H}$, weil $\mathrm{H}$ unter den Handlungsalternativen das geeignetste Mittel ist, das Ziel Q zu realisieren oder zu befördern. Das Erreichen dieses Ziels trägt zur größtmöglichen und langfristigen Verbesserung der erwarteten Situation bei. Unter den Alternativen, die der Bürgermeister hat, ist die Einrichtung des Gewerbegebiets tatsächlich die in dieser Situation größtmögliche Verbesserung. Die Gemeinde kann das Kindergartenprojekt finanzieren. Wir haben sichergestellt, dass sich der Bürgermeister in der Entscheidung für $\mathrm{H}$ nicht nur konform zu dem Primärgrund verhält (indem er $\mathrm{z}$. B. $\mathrm{H}$ wegen eines Bestechungsgeldes tut), sondern $\mathrm{H}$ tatsächlich um willen dieses Primärgrundes tut.

Wir können die Entscheidung des Bürgermeisters ohne zu zögern eine gute Entscheidung nennen. Aber ist sie auch weise? Ja, wenn wir nur auf das Resultat blicken. Wir haben uns gegenüber (WE-0) stark verbessert. Das Resultat ist nicht irgendwie zustande gekommen, sondern überlegt. Der Bürgermeister hat sich geleitet durch einen rechtfertigenden Grund entschieden.

Aber ist die Entscheidung auch auf weise Art zustande gekommen? Hier lautet die Antwort: Nein.

\section{WEISES ENTSCHEIDEN UND KOGNITIVE KONTROLLE}

In unserer Analyse finden sich noch dunkle Stellen, die aufgeklärt werden müssen. Was genau im Dunkeln geblieben ist, wird deutlich, wenn wir uns fragen, was in der Entscheidungsfindung noch hätte schieflaufen können.

Bisher sind wir von einem perfekten Primärgrund ausgegangen ebenso wie von Überzeugungen, die eindeutig $\mathrm{H}$ als das geeignetste Mittel ausweisen. Wie unsere Bürgermeister-Szenarien zeigen, ist dieses Bild zu simpel. Die Fälle, in denen sich aus der Menge alternativer Handlungsoptionen genau eine Option als perfekter rechtfertigender Grund aufdrängt, sind eher selten. In unseren BürgermeisterSzenarien sind es zwei sich ausschließende Handlungsoptionen, für die Gründe und Gegengründe erwogen werden müssen. Gerade in den komplexen Situationen, die eine weise Entscheidung erfordern, finden sich in der Menge der Gründe oft auch falsche Überzeugungen und unvertretbare Ziele, die meist nicht auf 
den ersten Blick als solche erkennbar sind. Was der Analyse also fehlt, ist eine Reflexion der Gründe, in der das Für und Wider unter Anlegung strenger epistemischer Maßstäbe geprüft wird. Wenn man den letzten abschließenden Schritt (WE-ref) in einen Slogan bringen will, würde er lauten:

Slogan: „Weise handelt, wer in einer schwierigen Situation überlegt und kontrolliert das Beste auf der Basis der richtigen Art von Gründen tut.“

Unser Bürgermeister hat nicht den einen perfekten Grund für seine Entscheidung. Vielmehr muss er sorgfältig überlegen, welche Gründe für das Gewerbegebiet und welche gegen das Gewerbegebiet sprechen. In der Abwägung Fördersumme gegen Erhaltung des Waldgebiets hat er pro tanto-Gründe, die vielleicht für die eine oder die andere Option sprechen. Er kann diese Gründe gewichten, um dann der Seite mit dem größeren Gesicht zu folgen.

Doch dieser Ansatz ist unzureichend. S muss nicht nur pro-tanto Gründe in der Abwägung einer Alternative berücksichtigen; die Anforderung an eine reflektierende Kontrolle über die Gründe sind viel höher: S muss alle in dieser Situation möglichen Optionen erfassen, gewichten und ausbalancieren, d. h. S muss sich auf ,all things considered reasons“ stützen, wenn er die Entscheidung rechtfertigen will. Im Beispielsfall kann sich $\mathrm{S}$ entweder für das Gewerbegebiet entscheiden, weil S:

G1: die Fördersumme zur Haushaltssanierung einstreichen will und/oder

G2: langfristig Gewerbeeinnahmen erzielen möchte und/oder

G3: das Kindergartenprojekt finanzieren will.

Oder $\mathrm{S}$ entscheidet sich dagegen, weil S:

GG1: das Waldgebiet zur Sicherung der Wohn- und Luftqualität erhalten will.

GG2: die Einnahmen aus dem Tourismus in der bisherigen Höhe halten will.

In diesem Deliberationsprozess kann einiges schieflaufen. Wieder hilft uns diese Art von Betrachtung dabei, die Definition zu verbessern:

1. Der Akteur S verfügt über keine ausreichende Urteilskompetenz:

Angenommen der Bürgermeister S hat die falsche Vorstellung, dass der Gemeindewald wegen möglicher Tollwut und Zecken eine Gesundheitsgefahr darstellt, oder dass sich Wölfe ansiedeln könnten oder gar, dass böse Dämonen dort wohnen. Wenn S solche Vorbehalte gegen den Wald hat, hat er dann die Alternative, den Wald nicht für das Gewerbegebiet zu opfern, überhaupt angemessen bewertet? Sind die Überzeugungen von S, obwohl falsch, wenigstens gerechtfertigt?

Es wäre zu billig, hier einfach in die externalistische Betrachtungsweise zu wechseln und von außen festzulegen, welche Informationen dem Bürgermeister fehlen und welche Überzeugungen über den Wald wahr sind. Die Kritik sollte die Sicht des handelnden Akteurs S einnehmen und aus dessen Perspektive erfolgen. Das heißt: Der für die Entscheidung relevante Informationsstand muss 
für S prinzipiell kognitiv erreichbar und zugänglich sein. Würden wir einen Maßstab von außen anlegen, müssten wir viele Personen, wie z. B. Homer, Sokrates, Aristoteles, Ptolemäus usw., die wir für weise halten, aus der Ehrengalerie der Weisen ausschließen. Sie alle hatten viele falsche, wenngleich gerechtfertigte Überzeugungen z. B. hinsichtlich der Relation der Erde zur Sonne. Sie lebten in einer Umgebung, in der die entsprechenden wahren Überzeugungen einfach kognitiv noch nicht zugänglich waren.

Im Fall des Bürgermeisters jedoch liegen die entsprechenden wahren Überzeugungen prinzipiell in seiner kognitiven Reichweite. Da er sich über die Risiken von Tollwut und Zecken kundig machen könnte und da er seine abergläubischen Vorstellungen korrigieren könnte, hat er nicht nur falsche Überzeugungen, er ist darin auch nicht gerechtfertigt. Wir erwarten von einem weisen Entscheidungsprozess nicht nur, dass der Akteur S eine große Bandbreite gerechtfertigter Überzeugungen auf den für die Entscheidung relevanten Gebieten besitzt, sondern überdies dass er diese im Hinblick auf neue Informationen ständig überprüft und ggf. durch tiefere und rationale Überzeugungen ersetzt. ${ }^{6}$ Damit verlangen wir nicht nur, dass sich S tadelsfrei verhält, d. h. keine epistemischen Pflichten verletzt, sondern dass $S$ sich lobenswürdig verhält. S sollte sich über die in der Entscheidungssituation unmittelbar verfügbaren Informationen hinaus um weitere (kognitiv zugängliche) Informationen bemühen, die die Entscheidung - all things considered - absichern.

Wohlgemerkt, wir verlangen von unserem Akteur hier nicht Wissen. (Wissen wäre wahre gerechtfertigte Überzeugung). Diese Bedingung wäre zu stark und würde weise Entscheidungen höchst selten machen. Es ist immer noch möglich, dass sich der Akteur irrt, obwohl er auf der Basis aller ihm zugänglichen Informationen gerechtfertigt ist. Deshalb ist es fruchtbarer im Zusammenhang mit Weisheit von Verstehensleistungen auszugehen und nicht von Wissen. ${ }^{7}$ Wir verlangen Urteilskompetenz nur im Sinne von gerechtfertigten und ständig überprüften Überzeugungen.

\section{Dem Akteur fehlt es an inferentieller Kompetenz:}

Die Reflexion als auf Gründe gerichteter Deliberationsprozess hängt auch von den inferentiellen Fähigkeiten des Akteurs ab. Der Bürgermeister muss seine Absichten und Überzeugungen abduktiv, deduktiv und induktiv korrekt mit den jeweiligen Gründen verbinden um zu dem richtigen Ergebnis zu kommen. Er ist epistemisch nicht nur wegen ungerechtfertigter Überzeugungen zu tadeln, sondern auch wenn er auf inkorrekte Weise Schlüsse zieht.

\footnotetext{
${ }^{6}$ Vgl. Ryan, „Wisdom,” 118.

${ }^{7} \mathrm{Zu}$ untersuchen, welche Art von Verstehensleistungen hier gefordert sind und wie sie sich von Wissen unterscheiden, wäre eine eigene Abhandlung.
} 


\section{Dem Akteur fehlt es an Variationskompetenz:}

Wenn der Bürgermeister überlegt, ob er das Gewerbegebiet einrichten soll oder nicht, muss er kontrafaktische Überlegungen über die Relation von Gründen und Zielen anstellen, wie z. B. „Was wäre, wenn das Gewerbegebiet nicht eingerichtet wird? Dann kann das Kindergartenprojekt nicht finanziert werden." Und: „Wenn der Wald nicht abgeholzt werden würde, bliebe die Wohn- und Luftqualität gut" usw. Ohne die Handlungsoptionen im Geiste zu manipulieren und die Folgen durchzuspielen, kommt der Akteur nicht zu einem kontrollierten Gesamtergebnis, d. h. er kommt nicht auf kontrollierte Weise zu dem Grund, der schlussendlich handlungswirksam werden soll. ${ }^{8}$

Unter Anwendung dieser Fähigkeiten gelangt der Bürgermeister - so wollen wir annehmen - zu dem Ergebnis, dass die Gründe G1 bis G3 schwerer wiegen als die Gegengründe GG1 und GG2. Die erwarteten Einnahmen sind höher als die erwarteten Verluste und zwar so viel höher, dass auch die Einbuße an Wohnqualität für die Einwohner ausgeglichen wird. Die Anforderungen an den Entscheidungsprozess können wir nun als kognitive Kontrolle über die Gründe festlegen:

(WE-ref)

(v) S (kraft Urteilskompetenz, inferentieller Kompetenz und Variationskompetenz) kognitive Kontrolle über die Gründe-liefernden Relationen ausübt (epistemische Kontrollbedingung).

Haben wir mit der Bedingung (v) endlich sichergestellt, dass aus einer - wie auch immer zustande gekommenen - guten Entscheidung ein weiser Entscheidungsprozess wird? Nein, es fehlt noch ein entscheidender Schritt.

\section{REFLEKTIERTES WEISES ENTSCHEIDEN}

Betrachten wir nochmals unseren Modellfall:

\section{(Bürgermeister-Szenario 1):}

1) Dem Bürgermeister wird aus EU-Mitteln eine sehr hohe Fördersumme für den insolvenzbedrohten Haushalt der Gemeinde angeboten, wenn er in seiner Gemeinde ein Gewerbegebiet einrichtet.

2) Gegen das Gewerbegebiet hat er starke Vorbehalte. Er wünscht sich aber, das Gewerbegebiet einrichten zu können (den Gemeinderat dazu zu bringen, die Einrichtung des Gewerbegebiets zu beschließen), um die Fördersumme zu erhalten.

3) Für das Gewerbegebiet spricht, dass langfristig Gewerbesteuereinnahmen zu erzielen sind, aus denen das lange geplante Kindergartenprojekt finanziert werden kann. ${ }^{8}$ Vgl. zu dieser Art kognitiver Kontrolle: Alison Hills, „Understanding-why,” Nous 50,
Nr. 4, (2015): 1-28. 
4) Für das Gewerbegebiet müsste aber ein nahes Waldgebiet abgeholzt werden, das entscheidend zur Wohn- und Luftqualität im Ort beiträgt. Die Abholzung würde zu Einbußen im Tourismus-Geschäft führen.

Ist die hohe Fördersumme tatsächlich ein Grund, der die Einrichtung des Gewerbegebiets rechtfertigt? Wenn ja, steht dieser Grund auf einer Stufe mit dem Gegengrund, der gegen das Gewerbegebiet spricht, nämlich auf einer Stufe mit dem Verlust der Wohn- und Luftqualität und der Einbuße im Tourismusgeschäft?

Der Bürgermeister kann sich entweder für das Gewerbegebiet entscheiden, weil er:

G1: die Fördersumme zur Haushaltssanierung einstreichen will und/oder

G2: langfristig Gewerbeeinnahmen erzielen möchte und/oder

G3: er das Kindergartenprojekt finanzieren will.

Oder er entscheidet sich dagegen, weil er:

GG1: das Waldgebiet zur Sicherung der Wohn- und Luftqualität erhalten will.

GG2: die Einnahmen aus dem Tourismus in der bisherigen Höhe halten will.

Die Frage mag Verwirrung stiften, ist doch die Einrichtung des Gewerbegebiets die Bedingung dafür, dass die Fördersumme zur Haushaltssanierung an die Gemeinde fließt. Wenn wir aber genauer hinsehen, wird klar: Die Gründe G2 und G3 sowie die beiden Gegengründe GG1 und GG2 werden durch Tatsachen über das Gewerbegebiet geliefert. Die Einrichtung des Gewebegebiets lässt Gewerbesteuern fließen und die Einrichtung des Gewerbegebiets führt zu einer Einbuße an Wohnqualität und Tourismus-Geschäft. Diese Gründe haben jedoch nichts mit dem Wunsch nach der Fördersumme zu tun. Es gibt diese Gründe als normative rechtfertigende Gründe, auch wenn die EU keine Fördersumme ausgeschrieben hätte.

Der Grund G1 dagegen hat etwas mit der konativen Einstellung des Bürgermeisters zu tun. Er muss - in seiner Perspektive - erwägen: wie lägen die Dinge, wenn ich die Fördersumme wünschen bzw. nicht wünschen würde, d. h. wenn ich die konative Pro-Einstellung dazu einnehmen bzw. nicht einnehmen würde.

Es ist also von zwei verschiedenstufigen konativen Pro-Einstellungen und entsprechend von zwei verschiedenartigen Gründen die Rede: ${ }^{9}$

\footnotetext{
${ }^{9}$ Bezugspunkt in solchen Überlegungen ist Parfits intensiv diskutierte und immer wieder modifizierte Unterscheidung zwischen object-given und state-given Gründen. Vgl. Derek Parfit, „Rationality and Reasons," in Exploring Practical Philosophy, hg. von Dan Egonsson et al. (Aldershot: Ashgate, 2001), 17-39.

Auf die Debatte kann hier nicht eingegangen werden. Mit Persson bin ich der Meinung, dass die Konzeption von verschiedenstufigen Gründen allen Einwänden gegen Parfits Unterscheidung standhält. Vgl. Ingmar Persson, „Primary and Secondary Reasons,” in Hommage á Wlodek. Philo-
} 
1. Primärer Grund für das Einnehmen einer erststufigen konativen ProEinstellung (Beabsichtigen, Wünschen usw.): S will / wünscht das Gewerbegebiet, weil G.

2. Sekundärer Grund für eine zweitstufige konative Pro-Einstellung zum Einnehmen von erststufigen konativen Pro-Einstellungen: S will / wünscht den Wunsch nach dem Gewerbegebiet zu haben (wünscht, dass der Gemeinderat das Gewerbegebiet will) weil G'.

In unserem Modellfall sind beide Pro-Einstellungen über eine Gründe-Relation verknüpft. Die begehrte Fördersumme erhält der Bürgermeister nur, wenn er wünscht, dass er bzw. der Gemeinderat das Gewerbegebiet wünscht. Der Bürgermeister hat einen Grund, die hohe Fördersumme zu wollen, und er hat einen Grund, das Gewerbegebiet zu wollen. In beiden Fällen scheint der Grund der Wunsch nach der hohen Fördersumme zu sein. $G$ scheint identisch mit $G^{6}$ zu sein. Doch unser Bürgermeister erliegt hier einer Konfusion. Das wird deutlicher wenn wir das Bürgermeister-Szenario nochmals modifizieren:

\section{(Bürgermeister-Szenario 3)}

1) Der Bürgermeister wird von der Mafia bedroht. Sie droht mit der Terrorisierung der Bevölkerung, wenn der Bürgermeister das Gewerbegebiet nicht einrichten will.

2) $\ldots$

3) $\ldots$

4) $\ldots$

Solche Szenarien werden als „Böse-Dämonen-Szenarien“ diskutiert. Ein böser Dämon droht mir schlimme Bestrafung an, wenn ich keine Pro-Einstellung zu einer Schale voll Dreck einnehme. Klarerweise habe ich einen Grund - nämlich die Schmerzvermeidung - eine Pro-Einstellung zu der Schale voll Dreck einzunehmen, z. B. sie für wertvoll zu halten. Ich will also aus dem Grund der Schmerzvermeidung mich dazu bringen, die Schale voll Dreck für gut zu halten. Die Schale voll Dreck ist natürlich im günstigsten Fall wertlos. Der Grund, der für die zweitstufige Pro-Einstellung spricht, nämlich zu wollen, dass ich die Schale voll Dreck für wertvoll halte, ist die Vermeidung der Bestrafung. Dieser Grund hat nichts mit der erststufigen Einstellung zu der Schale voll Dreck zu tun, die ich natürlich eklig finde. ${ }^{10} \mathrm{G}$ ist nicht mit $\mathrm{G}^{6}$ identisch.

sophical Papers Dedicated to Wlodek Rabinowicz, hg. von Toni Rønnow-Rasmussen et al. (Lund: Department of Philosophy, Lund University 2007).

${ }^{10}$ Vgl. dazu: Roger Crisp, „Review of Kupperman, Value ... and What Follows (by J. Kupperman)," Philosophy 75 (2000): 458-492; Wlodek Rabinowicz und Toni Rønnow-Rasmussen, „The Strike of the Demon: On Fitting Pro-attitudes and Value," Ethics 114 (2004): 391-423; Jonas Olson, „Buck-Passing and the Wrong Kind of Reasons,” The Philosophical Quarterly 54 (2004): 295-300; Philip Stratton-Lake, „How to Deal with Evil Demons: Comment on Rabinowicz and Rønnow-Rasmussen,” Ethics 115 (2005): 788-798. 
Zurück zu unserem Bürgermeister: Der Grund, den der Bürgermeister hat, die zweitstufige Einstellung einzunehmen, nämlich zu wünschen, dass der Gemeinderat das Gewerbegebiet will, hat nichts mit den Gründen zu tun, die für oder gegen das Gewerbegebiet selbst sprechen.

Die Gründe für oder gegen das Gewerbegebiet sind primäre Gründe; die Gründe, die Fördersumme zu erhalten (bzw. den Mafia-Terror zu vermeiden) sind sekundäre Gründe.

Was im Fall der Mafia-Drohung leicht auseinanderzuhalten ist, muss auch im positiven Fall des Angebots der hohen Fördersumme für den Haushalt getrennt werden: Der sekundäre Grund, der dafür spricht, eine zweitstufige ProEinstellung zu dem Ziel einzunehmen, das Gewerbegebiet einzurichten, ist ein anderer als der primäre Grund, der für die Einrichtung des Gewerbegebiets selbst spricht: nämlich Gewerbesteuern einzunehmen, das Kindergartenprojekt $\mathrm{zu}$ finanzieren usw. Und natürlich ist er auch etwas anderes als der primäre Grund, der dagegen spricht, z. B. dass der Wald abgeholzt werden muss usw.

Wer erststufige mit zweitstufigen Gründe konfundiert, hält fälschlicherweise folgende Prinzipien für gültig, die Ingmar Persson etwa so herausgearbeitet hat: ${ }^{11}$

(Abwärts) Wenn $\mathrm{G}$ für $\mathrm{S}$ ein Grund ist, eine zweitstufige konative ProEinstellung zu Q einzunehmen (zu wünschen, dass $S$ wünscht dass $Q$ ), dann ist $\mathrm{G}$ auch ein Grund, eine erststufige Pro-Einstellung zu Q einzunehmen ( $\mathrm{Q} z u$ wünschen).

(Aufwärts) Wenn $\mathrm{G}$ für $\mathrm{S}$ ein Grund ist, eine erststufige konative ProEinstellung zu Q einzunehmen, dann ist $\mathrm{G}$ auch ein Grund, eine zweitstufige Pro-Einstellung zu der erststufigen Pro-Einstellung einzunehmen.

Beide Prinzipien sind falsch. Gegen (Aufwärts) spricht, dass wir gelegentlich Wünsche haben, die wir nicht haben wollen. H. Frankfurt hat dafür Beispiele genannt, wie der Fall des Drogensüchtigen wider Willen. Wir können uns in dieser Frage auch an einen Klassiker halten: ${ }^{12}$ Kant meint, dass wir keine guten Gründe für erststufige konative Pro-Einstellungen zu Ehre, Macht, Geld haben. Sie arten leicht zu den Untugenden der Ehrsucht, Herrschsucht, Habsucht aus. Stattdessen sollten wir lieber nach Demut, Friedfertigkeit und Bescheidenheit streben.

Wir haben aber gute Gründe für zweitstufige Pro-Einstellungen zu solchen erststufigen Pro-Einstellungen: Es ist Kant zufolge der Wettstreit, die ungesellige Geselligkeit, durch die die Natur den Menschen zur Kultur treibt. Wir haben einen guten sekundären Grund, nämlich die Kulturförderlichkeit, wenn wir wol-

\footnotetext{
${ }^{11}$ Vgl. dazu Persson, „Primary”, ebd.

12 Vgl. dazu Gerhard Schönrich, „Kants Werttheorie? Versuch einer Rekonstruktion,” KantStudien 104 (2013): 321-345.
} 
len, dass die Akteure nach Ehre, Macht und Geld streben. Das Gegenbild, nämlich ein ,arkadisches Schäferleben bei vollkommener Eintracht, Genügsamkeit und Wechselliebe“ würde eben nicht jenen Stachel bieten, der die „Talente nach und nach entwickelt". Die arkadische Existenz hätte, wie Kant bissig anmerkt, nicht mehr Wert als das Dasein des Hausviehs (Idee zu einer allgemeinen Geschichte, AA 08: 21). Wir sitzen einer ,inneren praktischen Täuschung“ auf, einem „Wahn“ (Anthropologie, AA 07: 274), wenn wir glauben, gute primäre Gründe für das Streben nach Ehre, Macht und Geld zu haben. Die Natur, so Kant, spiegelt dem grundsätzlich eher faulen Menschen Ehre, Macht und Geld als primär gut begründetes Streben vor. Der Philosoph - der Freund der Weisheit - durchschaut diese Täuschung als eine List der Natur, die den Menschen so zu einem Kulturwesen befördert, wohl wissend, dass es sekundäre Gründe sind, die für die entsprechenden Einstellungen sprechen und nicht primäre.

Eine Entscheidung, die auf einer ,inneren praktischen Täuschung“ beruht wie im Falle unseres Bürgermeisters, kann keine weise Entscheidung sein. Wenn sich der Bürgermeister verleiten lässt, das Gewerbegebiet tatsächlich wegen des hohen Haushaltszuschusses zu wünschen, und er trotz dieser Konfusion $\mathrm{zu}$ einer guten Entscheidung kommt, hat er in diesem Szenario einfach nur Glück gehabt. Tatsächlich sprechen ja mehr Gründe für das Gewerbegebiet als dagegen. Diese primären Gründe haben aber nichts mit dem sekundären Grund zu tun, zu wünschen, dass er wünscht (oder der Gemeinderat wünschen soll), das Gewerbegebiet einzurichten. Es wäre ein „Wahn“ zu glauben, die Gründe für den Haushaltszuschuss oder die Drohung der Mafia hätten etwas mit den Gründen zu tun, die für oder gegen das Gewerbegebiet sprechen.

Damit haben wir nicht gesagt, dass der Bürgermeister zweitstufige Gründe ignorieren soll. Wir behaupten nur: Eine Entscheidung, die einer solchen Täuschung aufsitzt, ist keine weise Entscheidung. Solange der Bürgermeister diese Reflexion nicht anstrengt, kann er nicht weise entscheiden - auch wenn das Ergebnis für die Gemeinde gut ist. Egal wie die Entscheidung ausfällt, eine notwendige Bedingung für weises Entscheiden ist, dass sich die kognitive Kontrolle über Gründe auch auf die Frage erstreckt, welcher Ebene die Gründe angehören. Sind es primäre oder sekundäre Gründe? Sie (abwärts oder aufwärts) zu verwechseln bedeutet, sich auf die falsche Art von Gründen zu stützen. Deshalb fordern wir:

(vi) S die richtige Art von Gründen von der falschen Art unterscheidet (epistemische Meta-Kontrollbedingung).

Wenn wir die Fäden zusammenziehen und die Erfordernisse in ein Schema fassen, ergibt sich ein recht kompliziertes Geflecht von notwendigen Bedingungen. Aber etwas Einfacheres war auch gar nicht zu erwarten: 
(WE-0) $\mathrm{S}$ entscheidet sich in Situation $\mathrm{C}$ weise, $\mathrm{H}$ zu tun gdw.

(i) $\mathrm{H}$ unter den Handlungsalternativen, die $\mathrm{S}$ in $\mathrm{C}$ zur Verfügung stehen, das geeignetste Mittel ist, das zur Erreichung des Ziels Q, nämlich zur größtmöglichen und langfristigen Verbesserung der zu erwartenden Situation $\mathrm{C}^{*}$ beiträgt (qualitative Bedingung).

(WE-min)

(ii) $\mathrm{S}$ beabsichtigt $\mathrm{Q}$ zu realisieren und $\mathrm{S}$ die Überzeugung hat, dass $\mathrm{H}$ das geeignetste Mittel ist, Q zu erreichen (internalistische Handlungsbedingung).

(iii) das Ziel-Überzeugungs-Paar nach (ii) der rechtfertigende Primärgrund G für S's H-en ist (Rechtfertigungs-Bedingung).

(iv) S H aus dem Primärgrund G ausführt (starke Rationalitätsbedingung). (WE-ref)

(v) S (kraft Urteilskompetenz, inferentieller Kompetenz und Manipulationskompetenz) kognitive Kontrolle über die Gründe-liefernden Relationen ausübt (epistemische Kontrollbedingung).

(vi) S die richtige Art von Gründen von der falschen Art unterscheidet (epistemische Meta-Kontrollbedingung).

\section{BIBLIOGRAPHIE}

Cicero. Tusculanae Disputationes III. Hg. von Max Pohlenz. Leipzig: Teubner, 1918

Crisp Roger. „Review of Kupperman, Value ... and What Follows (by J. Kupperman).” Philosophy 75 (2000): 458-492.

Glück, Judith. Weisheit. Die fünf Prinzipien des gelingenden Lebens. München: Kösel-Verlag, 2016.

Heckhausen, Heinz und Gollwitzer, Peter M. „Thought Contents and Cognitive Functioning in Motivational versus Volitional States of Mind." Motivation and Emotion 11, Nr. 2 (1987), 101-120.

Hills Alison. „Understanding-why.” Nous 50, Nr. 4 (2015): 1-28.

Horn, Christoph, Antike Lebenskunst. Glück und Moral von Sokrates bis zu den Neuplatonikern. München: C. H. Beck, 1998.

Olson, Jonas. „Buck-Passing and the Wrong Kind of Reasons.” The Philosophical Quarterly 54 (2004): 295-300.

Parfit, Derek. „Rationality and Reasons.” In Exploring Practical Philosophy. Herausgegeben von Dan Egonsson, Jonas Josefsson, Bjorn Petersson, and Toni Rønnow-Rasmussen, 17-39. Aldershot: Ashgate, 2001.

Persson, Ingmar. „Primary and Secondary Reasons.” In Hommage á Wlodek. Philosophical Papers Dedicated to Wlodek Rabinowicz. Herausgegeben von Toni Ronnow-Rasmussen, Bjorn Petersson, Jonas Josefsson, and Dan Egonsson, Lund: Department of Philosophy, Lund University 2007.

Rabinowicz, Wlodek und Rønnow-Rasmussen Toni. „The Strike of the Demon: On Fitting Proattitudes and Value." Ethics 114 (2004): 391-423.

Ryan, Sharon. „Wisdom, Knowledge and Rationality.” Acta Analytica 27, Nr. 2 (2012): 99-112.

Schönrich, Gerhard. „Kants Werttheorie? Versuch einer Rekonstruktion.” Kant-Studien 104 (2013): 321-345. 
Speer, Andreas. „Weisheit.” In Historisches Wörterbuch für Philosophie, hg. von Joachim Ritter, Bd. 12. Basel: Schwabe AG 1971.

Stratton-Lake, Philip. „How to Deal with Evil Demons: Comment on Rabinowicz and RønnowRasmussen." Ethics 115 (2005): 788-798.

\section{WHAT MAKES DECISIONS WISE?}

It is methodologically easier to gain access to the concept of wise decisions than to the general concept of wisdom. Once we know what wise decisions are, the corresponding properties and abilities of the deciding person can be inferred. This paper will show that the analysis of wise decisions must not be restricted to the attributive reading of "S makes a wise decision", but must be extended to the adverbial reading of "S decides wisely". The externalistic perspective of the former reading exposes a necessary qualitative requirement (a long-range improvement of the situation for all parties involved) which must be met by the outcome of any wise decision. Only the internalistic perspective of the latter reading complies with our intuitions that the outcome must not be achieved by chance, but by the agent's epistemic abilities such as his competence of judging and reflecting the reasons for the decision. These abilities include in particular the agent's meta-competence of distinguishing first-order and second-order reasons that pertain not to the value of the object of the decision but rather to the value of the attitude underlying the decision.

\section{Keywords:}

wisdom, decision, reasons, value 